\title{
Epidemiology of migraine in men: Results from the Chronic Migraine Epidemiology and Outcomes (CaMEO) Study
}

Cephalalgia

2019, Vol. 39(2) 296-305

(C) International Headache Society 2018

Article reuse guidelines:

sagepub.com/journals-permissions DOI: $10.1177 / 0333102418786266$ journals.sagepub.com/home/cep

@SAGE

\author{
Ann I Scher', Shuu-Jiun Wang ${ }^{2,3}$, Zaza Katsarava ${ }^{4}$, \\ Dawn C Buse ${ }^{5,6}$, Kristina M Fanning ${ }^{7}$, Aubrey Manack Adams ${ }^{8}$ \\ and Richard B Lipton ${ }^{5,6,9}$
}

\begin{abstract}
Objective: To assess migraine epidemiology in men by examining gender differences in disease presentation, comorbidities, and prognosis.

Patients and methods: The Chronic Migraine Epidemiology and Outcomes (CaMEO) Study is a longitudinal survey of US adults with migraine identified by web questionnaire. Data were stratified by gender, collected between September 2012-November 2013, and included sociodemographics, headache features, Migraine Disability Assessment, Migraine Symptom Severity Score, Allodynia Symptom Checklist, and comorbidities. Discrete time hazard models addressed I-year likelihood of transition from episodic to chronic migraine headache frequency.

Results: Of the 16,789 migraine respondents, 4294 were men (25.6\%). Compared to women, men were slightly older at onset of their headaches (mean 24.I vs. 22.3 years) and had fewer headache days/month (4.3 vs. 5.3 days), slightly less severe attacks (Migraine Symptom Severity Score, 21.6 vs. 22.6), reduced frequencies of grade IV Migraine Disability Assessment scores (I $5.7 \%$ vs. $24.1 \%)$, allodynia (32.6\% vs. $49.7 \%)$, chronic migraine $(6.5 \%$ vs. $9.6 \%$, each $p<0.001)$, and common comorbidities. Men were less likely to report consulting a doctor for their headaches and receiving a migraine diagnosis if they consulted. Men and women with episodic migraine had similar crude I-year risk of chronic migraine onset. Controlling for known risk factors (i.e. depression, headache frequency, allodynia), men had greater likelihood of chronic migraine onset at 6, 9, and 12 months (each $p<0.05$ ).

Conclusions: Findings confirmed gender differences. Men with migraine generally have less severe attacks and disability and are less likely to receive a diagnosis than women with migraine. Prognostic factors may be better understood for women than men.
\end{abstract}

\section{Keywords}

CaMEO, chronic migraine, disability, headache frequency, epidemiology, episodic migraine, men

Date received: 29 January 2018; revised: 16 May 2018; accepted: I June 2018

\section{Introduction}

Migraine is a common, disabling disease that affects men and women, with an estimated global prevalence in 2010 of approximately $11 \%$ in men and $19 \%$ in

'Department of Preventive Medicine and Biometrics, affiliated with Uniformed Services University of Health Sciences, Bethesda, MD, USA

${ }^{2}$ Faculty of Medicine, National Yang-Ming University School of Medicine, Taipei, Taiwan

${ }^{3}$ Department of Neurology, Neurological Institute, Taipei Veterans Hospital, Taipei, Taiwan
${ }^{4}$ Evangelical Hospital Unna, Department of Neurology, University of Essen, Essen, Germany

${ }^{5}$ Department of Neurology, Albert Einstein College of Medicine, Bronx, NY, USA

${ }^{6}$ Montefiore Medical Center, Bronx, NY, USA

${ }^{7}$ Vedanta Research, Chapel Hill, NC, USA

${ }^{8}$ Allergan plc, Irvine, CA, USA

${ }^{9}$ Department of Epidemiology and Population Health, Albert Einstein College of Medicine, Bronx, NY, USA

Corresponding author:

Ann I Scher, Department of Preventive Medicine and Biometrics, Uniformed Services University of the Health Sciences, 430 I Jones Bridge Rd, Bethesda, MD 208I4-4799, USA.

Email: ann.scher@usuhs.edu 
women (1). In 2015, the Global Burden of Disease Study ranked migraine as the fourth leading cause of years lived with disability in women and the eighth leading cause in men $(2,3)$. The established higher prevalence of migraine in women (4-7), as well as differences in consulting patterns (8), may have led to an under-appreciation of the impact and presentation of migraine in men.

The Chronic Migraine Epidemiology and Outcomes (CaMEO) Study is a longitudinal study embedded with cross-sectional modules. It was designed to be representative of the US migraine population and to explore the course of migraine (9), potential barriers to effective diagnosis and treatment (10), novel aspects of disease burden (11), and patterns of comorbidity $(12,13)$. These data were analyzed to gain a better understanding of gender differences related to the presentation and prognosis of migraine. To elucidate the epidemiology of migraine in men, this analysis reports gender differences in migraine presentation, comorbid conditions, and short-term prognosis. An improved understanding of the epidemiology and patterns of consultation, diagnosis, and treatment of migraine in men may identify barriers to care and knowledge gaps related to the migraine phenotype in men.

\section{Methods}

\section{Study design}

The CaMEO Study, which has been published in full (12), consisted of a screening and recruiting phase to acquire a sample representative of the US population (9). This was followed by longitudinal measures conducted approximately every three months for one year embedded with web-based cross-sectional surveys to assess migraine symptoms and severity, headache-day frequency, headache-related disability, consulting and healthcare use, comorbid conditions, and family-related burden associated with headache, among other data.

\section{Participants}

The CaMEO Study data were collected over 15 months (September 2012 to November 2013) from a web-based panel consisting of 489,537 Research Now (Plano, TX) members. Of these individuals, 80,783 (16.5\%) responded and 58,418 (72.3\% of respondents) provided usable surveys for analysis; 16,789 (28.7\% of respondents with usable data) met the inclusion criteria, including meeting American Migraine Study/American Migraine Prevalence and Prevention (AMPP) Study diagnostic module criteria for migraine $(7,14)$, a modification of the International Classification of Headache Disorders, 3rd edition beta version (ICHD-3b) migraine criteria (15). Migraine respondents were stratified into episodic (EM) or chronic migraine (CM). $\mathrm{CM}$ classification was derived from Silberstein-Lipton criteria $(16,17)$ and ICHD-3b CM criteria. CM was defined as $\geq 15$ headache days/month averaged over the previous 3 months $(n=1476)$. EM was defined as $<15$ headache days/month averaged over the previous 3 months $(\mathrm{n}=15,313)$ (Supplemental Figure 1).

The web-based panel was constructed to be representative of the US population in terms of a number of demographic variables (age, gender, income, and geographic region). Participation rates in the CaMEO study were modest $(16.5 \%)$; to assess for selection bias, the respondents were compared with non-respondents across a number of demographic variables (e.g. age, gender, race, and markers of socioeconomic status) (12). Further, comparison of the AMPP and CaMEO Studies, both longitudinal cohort studies using different methods to evaluate migraine, revealed similar results for demographics, headache-related disability, and headache-day frequency, suggesting that the study populations were representative of and generalizable to the US migraine population (9).

\section{Primary assessments}

Measures included the following assessments: Age of severe headache onset, symptom severity (Migraine Symptom Severity Scale [MSSS]), disability (Migraine Disability Assessment [MIDAS]), anxiety symptoms (seven-item Generalized Anxiety Disorder scale [GAD-7]), depression symptoms (nine-item Patient Health Questionnaire [PHQ-9]), whether consulting a provider and the type of provider consulted (headache or non-headache specialist), acute prescription and over-the-counter (OTC) medication use, preventive prescription medication use, presence of aura, monthly headache-day frequency, body mass index (BMI), selfreported physician diagnosis of headache, headache management strategies, cutaneous allodynia (12-item Allodynia Symptom Checklist [ASC-12]), headache triggers, and comorbid conditions.

Headache disability was assessed using MIDAS, a five-item scale assessing missed and reduced productivity days at work, school, or home during the previous three months due to headache. Items were summed; scores $\geq 11$ indicated moderate-to-severe disability (grades III and IV) due to headache. MIDAS also assessed the number of headache days during the previous 3 months, which estimated monthly headache day frequency.

The MSSS sums responses to questions of how frequently each of the International Classification of Headache Disorders, 2nd edition (ICHD-2) criteria headache symptoms (unilateral pain; pulsating pain; 
moderate-to-severe pain; pain made worse by routine activities; feelings of nausea and/or vomiting; photophobia; and phonophobia) (18) are experienced with the most severe headache. Response options were coded as never (1), rarely (2), $<50 \%$ of the time (3), and $\geq 50 \%$ of the time (4), yielding a sum score from 7 to 28 .

The GAD-7 provides a measure of GAD severity during the preceding 2 weeks based on the scores determined for seven questions from 0 (not at all) to 3 (nearly every day) for a total severity score from 0 to 21 (moderate: 10-14; severe: 15-21). Respondents with a score $\geq 10$ were classified as having GAD.

The PHQ-9 assesses symptoms and functional impairment of the nine diagnostic criteria for depressive disorders in the preceding 2 weeks on a four-point (0-3) scale from 0 (not at all) to 3 (nearly every day). Depression was coded as a dichotomous variable using a cut score $\geq 10$, which indicated moderate (or worse) depressive symptomology.

Cutaneous allodynia during a headache was measured using the ASC-12, which rates responses for 12 symptoms as 0 (never or rarely), 1 ( $<50 \%$ of the time), or 2 ( $\geq 50 \%$ of the time). A score $\geq 3$ indicated the presence of allodynia $(19,20)$.

\section{Statistical analysis}

Headache features, disability, comorbidities, and prognosis were analyzed by gender. The chi-square test was used to identify significant gender differences for dichotomous or categorical variables. Independentgroup $t$ tests were used to evaluate differences for continuous variables.

Incidence and predictors of $\mathrm{CM}$ onset (e.g. reporting $\geq 15$ headache days/month at $\geq 1$ follow-up interviews) were evaluated using a discrete-time logit-hazard model. This model estimates the overall incidence and timing of $\mathrm{CM}$ onset and predictive factors, including gender, expressed as hazard ratios (HRs). This analysis was conducted among the subsample of respondents with EM at the baseline interview who completed $\geq 1$ follow-up survey offered at 3, 6, 9, and 12 months after the initial baseline survey $(n=9307)$.

Respondents were included in this prognostic analysis only for the follow-up period(s) for which they had complete data. Respondents could miss $\geq 1$ follow-up period and be included in future periods. The longitudinal analysis by necessity excluded those lost to follow-up. To consider the possibility that observed gender effects could be associated with loss to followup (e.g. informative censoring), we conducted separate binary logistic regression models for each follow-up period to evaluate whether known likely prognostic baseline factors for CM onset (e.g. anxiety, depression, headache frequency, allodynia) predicted loss to followup and whether loss to follow-up was equally likely in men and women. The models were first run separately for men and women, and then an additional model including the interaction of gender with each of the four comorbid conditions was calculated. Two-tailed $p$ values $<0.05$ were considered statistically significant. Descriptive analyses and binary logistic models were conducted with IBM SPSS Statistics, version 20.0 (IBM, Armonk, NY; 2011). Discrete-time logit-hazard modeling (GLM with a logit link, applied to the person-period data set) was performed using $\mathrm{R}$, version 2.14; the preparation of the person-period data set used for this modeling was done with the help of the R packages reshape2 and dplyr (21).

\section{Results}

\section{Demographics and baseline characteristics}

Of the 16,789 respondents who met the criteria for migraine, 4294 were men (25.6\%). Compared with women, men were older ( 42.0 vs. 40.8 years, $p<0.001$ ); had an older age of severe headache onset ( 24.1 vs. 22.3 years, $p<0.001)$; were more likely to be Caucasians $(86.3 \%$ vs. $83.1 \%, p<0.001)$; had a larger proportion with annual household income $\geq \$ 50,000(64.7 \%$ vs. $57.8 \%, p<0.001$ ); and were more likely to be overweight/obese $(73.4 \%$ vs. $60.2 \%, p<0.001$; Table 1$)$. A smaller proportion of men than women were classified as CM $(6.5 \%$ vs. $9.6 \%, p<0.001)$. Men had fewer monthly headache days (4.3 vs. $5.3, p<0.001)$, lower MSSS scores (21.6 vs. 22.6, $p<0.001)$, and were less likely to have MIDAS scores in the moderate or severe range (grade III, $13.9 \%$ vs. $18.1 \%$; and grade IV, $15.7 \%$ vs. $24.1 \%, p<0.001)$, allodynia $(32.6 \%$ vs. $49.7 \%, p<0.001)$, and aura symptoms $(41.3 \%$ vs. $49.3 \%, p<0.001$; Table 2).

\section{Comorbid conditions, doctor consultation profiles, and headache triggers in men versus women}

Comorbid conditions that were more prevalent in men with migraine included emphysema, heart disease/ angina, hypertension, and stroke; those that were more common in women included allergies, asthma, bronchitis, sinusitis, anxiety, temporomandibular disorder, and circulation problems such as Raynaud syndrome, fainting, heart attack, and irregular heartbeat, among others (all $p<0.05$; Figure 1(a)). Men were less likely than women to have reported ever consulting a doctor to manage their headaches ( $70.2 \%$ vs. $77.2 \%, p<0.001)$, to have recently consulted a doctor to manage their headaches $(28.6 \%$ vs. $31.1 \%, p<0.001)$, to have received a diagnosis of migraine or $\mathrm{CM}$ if they ever 
Table I. Sociodemographic characteristics at baseline by gender among persons with episodic and chronic migraine.

\begin{tabular}{|c|c|c|c|c|}
\hline & $\begin{array}{l}\text { Men } \\
(n=4294)\end{array}$ & $\begin{array}{l}\text { Women } \\
(n=12,495)\end{array}$ & Estimate & $p$-value \\
\hline Age (years), mean (SD) & $42.0(15.3)$ & $40.8(14.1)$ & $1.13(0.63-1.63)^{\mathrm{a}}$ & $<0.001$ \\
\hline $\begin{array}{l}\text { Age of onset of most severe } \\
\text { headache type (years), mean (SD) }\end{array}$ & $24.1(13.2)$ & $22.3(10.9)$ & $1.84(1.44-2.25)^{\mathrm{a}}$ & $<0.001$ \\
\hline Caucasian, n (\%) & $3698(86.3)$ & $10,346(83.1)$ & $24.1^{\mathrm{b}}$ & $<0.001$ \\
\hline Employed full- or part-time, $\mathrm{n}(\%)$ & $2818(65.6)$ & $8126(65.0)$ & $0.5^{\mathrm{b}}$ & 0.48 \\
\hline Education $\geq 4$-year college degree, $n(\%)$ & $1985(46.2)$ & $5562(44.5)$ & $3.8^{\mathrm{b}}$ & 0.052 \\
\hline Household income $\geq \$ 50,000, n(\%)$ & $2768(64.7)$ & $7164(57.8)$ & $62.2^{\mathrm{b}}$ & $<0.001$ \\
\hline With insurance coverage, n (\%) & $3563(86.0)$ & $10,584(86.9)$ & $2.1^{\mathrm{b}}$ & 0.15 \\
\hline Overweight/obese, n (\%) & $3150(73.4)$ & $7520(60.2)$ & $239.5^{b}$ & $<0.001$ \\
\hline
\end{tabular}

Note: Percentages are based on number of respondents, which varied for each item.

aPoint estimate is mean difference and $95 \% \mathrm{Cl}$.

${ }^{b}$ Point estimate is chi-square.

Table 2. Headache characteristics and management at baseline by gender among men and women with episodic or chronic migraine.

\begin{tabular}{|c|c|c|c|c|}
\hline & $\begin{array}{l}\text { Men } \\
(n=4294)\end{array}$ & $\begin{array}{l}\text { Women } \\
(n=12,495)\end{array}$ & Estimate & $p$-value \\
\hline \multicolumn{5}{|l|}{ Migraine status, n (\%) } \\
\hline Episodic & $4015(93.5)$ & II,298 (90.4) & $37.9^{\mathrm{a}}$ & $<0.001$ \\
\hline Chronic & $279(6.5)$ & $1197(9.6)$ & & \\
\hline $\begin{array}{l}\text { Headache-day frequency } \\
\text { (days/month), mean (SD) }\end{array}$ & $4.3(5.6)$ & $5.3(6.1)$ & $-1.03(-1.24 \text { to }-0.82)^{c}$ & $<0.001$ \\
\hline $\mathrm{MSSS}^{\mathrm{b}}$, mean $(\mathrm{SD})$ & $21.6(3.1)$ & $22.6(3.2)$ & $-1.04(-1.15 \text { to }-0.93)^{c}$ & $<0.001$ \\
\hline \multicolumn{5}{|l|}{ MIDAS category, n (\%) } \\
\hline Little/no disability (grade I, score 0-5) & $2352(54.8)$ & $5018(40.2)$ & $299.1^{\mathrm{a}}$ & $<0.001$ \\
\hline Mild disability (grade II, score 6-I0) & $672(15.7)$ & $2202(17.6)$ & & \\
\hline Moderate disability (grade III, score II-20) & $597(13.9)$ & $2266(18.1)$ & & \\
\hline Severe disability (grade IV, score $\geq 2 \mathrm{I}$ ) & $672(15.7)$ & $3004(24.1)$ & & \\
\hline Allodynia, n (\%) & $105 \mid(32.6)$ & 4769 (49.7) & $284.0^{\mathrm{a}}$ & $<0.001$ \\
\hline \multicolumn{5}{|l|}{ Visual aura symptoms, $\mathrm{n}(\%)^{\mathrm{d}}$} \\
\hline None & $2521(58.7)$ & $6340(50.7)$ & $109.2^{\mathrm{a}}$ & $<0.001$ \\
\hline Positive symptoms only (e.g. spots, stars) & $1349(31.4)$ & $4684(37.5)$ & & \\
\hline Negative symptoms only (e.g. vision loss) & $213(5.0)$ & $521(4.2)$ & & \\
\hline Positive and negative symptoms & $211(4.9)$ & $950(7.6)$ & & \\
\hline Self-reported triggers, n (\%) & $1818(56.5)$ & $6575(68.6)$ & $156.3^{\mathrm{a}}$ & $<0.001$ \\
\hline $\begin{array}{c}\text { Number of triggers per } \\
\text { person, mean (SD) }\end{array}$ & $5.6(3.1)$ & $7.0(3.4)$ & $-1.46(-1.63,-1.29)^{c}$ & $<0.001$ \\
\hline
\end{tabular}

MIDAS: Migraine Disability Assessment; MSSS: Migraine Symptom Severity score.

Note: Percentages are based on number of respondents, which varied for each item.

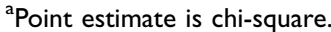

${ }^{\mathrm{b}}$ Total score range, 7-28; higher scores indicate greater severity.

'Point estimate is mean difference and $95 \% \mathrm{Cl}$.

d،Have you ever had vision changes just before or with any headache?" Positive: Spots, stars, lines, flashing lights, zigzag lines, or "heat waves." Negative: Loss of vision or partial loss of vision before headache. 


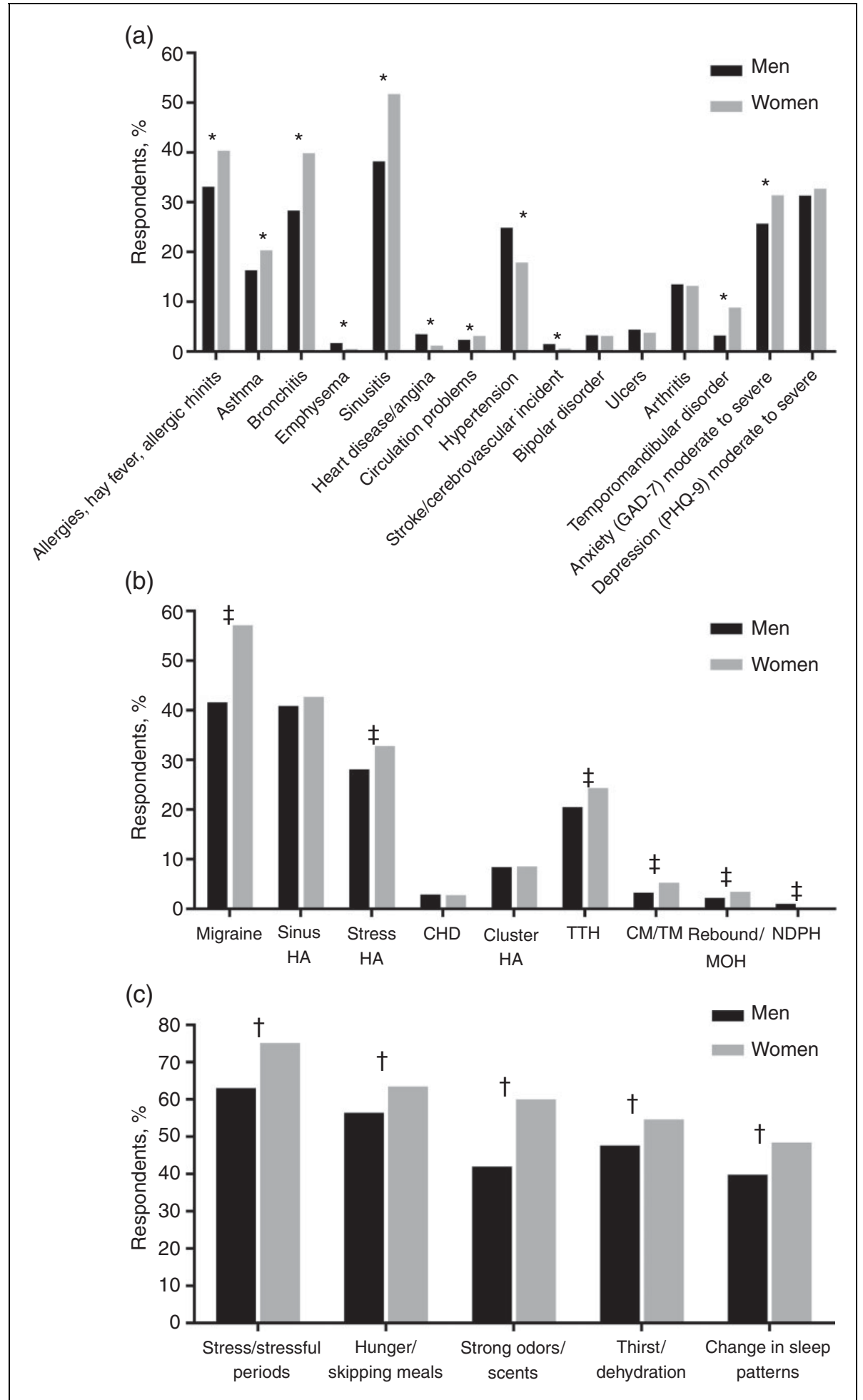

Figure I. Comorbidities (a), self-reported diagnoses by a healthcare professional (b), and perceived triggers of headache (c) stratified by gender. Response rates are reported for "yes" responses to a positive diagnosis from a healthcare professional, multiple diagnoses were possible, or to the presence of symptoms.

${ }^{*} p<0.05 ;{ }^{\dagger} p<0.01 ;{ }^{\star} p<0.001$.

$\mathrm{CDH}$ : chronic daily headache; CM: chronic migraine; GAD-7: seven-item Generalized Anxiety Disorder scale; $\mathrm{HA}$ : headache; $\mathrm{MOH}$ : medication overuse headache; NDPH: new daily persistent headache; PHQ-9: nine-item Patient Health Questionnaire; TM: transformed migraine; TTH: tension-type headache. 
Table 3. Baseline consulting and treatment patterns at baseline by gender among persons with episodic or chronic migraine.

\begin{tabular}{|c|c|c|c|c|}
\hline & $\begin{array}{l}\text { Men } \\
(n=4294)\end{array}$ & $\begin{array}{l}\text { Women } \\
(n=12,495)\end{array}$ & Estimate $^{\mathrm{a}}$ & $p$-value \\
\hline Ever consulted a doctor for a headache, $\mathrm{n}(\%)$ & $3014(70.2)$ & $9647(77.2)$ & 84.8 & $<0.001$ \\
\hline Currently consulting doctor for management, n (\%) & $863(28.6)$ & $3003(31.1)$ & 6.7 & $<0.01$ \\
\hline Diagnosed with migraine, $\mathrm{n}(\%)^{\mathrm{b}}$ & $1253(4 \mid .9)$ & $5504(57.5)$ & 223.8 & $<0.001$ \\
\hline \multicolumn{5}{|l|}{ Headache treatment use, n (\%) } \\
\hline None (no OTC or acute/preventive prescription) & $614(14.3)$ & $867(6.9)$ & 267.5 & $<0.001$ \\
\hline OTC only (no acute/preventive prescription) & $2328(54.2)$ & $6980(55.9)$ & & \\
\hline Acute prescription (no preventive, with/without OTC) ${ }^{c}$ & $797(18.6)$ & $3149(25.2)$ & & \\
\hline Preventive prescription (no acute, with/without OTC) & $184(4.3)$ & $408(3.3)$ & & \\
\hline Acute and preventive (with/without OTC) ${ }^{c}$ & $37 \mid(8.6)$ & $1091(8.7)$ & & \\
\hline
\end{tabular}

MSSS: Migraine Symptom Severity score; OTC: over-the-counter.

Note: Percentages are based on number of respondents, which varied for each item.

${ }^{\text {ap }}$ oint estimate is chi-square.

${ }^{b}$ Among those consulting.

'Includes opioids and barbiturates.

consulted a doctor $(41.9 \%$ vs. $57.5 \%, p<0.001)$, or to have used any prescription or OTC medication for headaches $(85.7 \%$ vs. $93.1 \%, p<0.001$; Table 3$)$. For most headache types, women were more likely to report a healthcare professional diagnosis (Figure 1(b)). Headache triggers were reported by $56.5 \%$ of men and $68.6 \%$ of women, among which the five most commonly reported triggers were stress/stressful times, hunger/skipping meals, strong odors/scents, thirst/dehydration, and change in sleep patterns (Figure 1(c)). Of all reported triggers, nine showed a predominance $>5 \%$ in women compared with men (hunger/skipping meals, thirst/dehydration, flashing/bright lights, extended screen exposure, neck strain, change in sleep patterns, caffeine[or lack of it], certain foods, changes in weather/temperature) and two showed a predominance $>10 \%$ (strong odors/ scents, stress/stressful times) (Supplementary Figure 2).

\section{Transition from episodic to chronic migraine in men versus women}

Approximately $3.4 \%$ (2.7\% men, $3.6 \%$ women) of the baseline EM population met the criteria for CM at 3month follow-up. Among those who had not met CM criteria in an earlier period, an additional $2.3 \%(2.4 \%$ men, $2.3 \%$ women) progressed to $\mathrm{CM}$ at 6 months, $1.7 \%(2.3 \%$ men, $1.5 \%$ women $)$ at 9 months and $1.8 \%$ (2.3 men, $1.6 \%$ women) at 12 months. This crude incidence of $\mathrm{CM}$ was similar for women and men at all time periods $(p>0.05)$. Controlling for demographics, headache features, and comorbidities, men have a $43 \%$ increase in the odds of CM onset across all follow-up periods (HR 1.43, 95\% CI $1.17-$ 1.73, Supplemental Table 1). Since two variables in the model (gender and MIDAS) violated the proportional hazard assumption, a final model was generated that included interactions for these variables by follow-up period. This final model confirmed that $\mathrm{CM}$ onset rates for gender and MIDAS were not consistent across the four follow-up periods. In the 3-month follow-up, the discrete-time hazard ratios of CM onset were similar for men and women $(p>0.05)$. In the 6-month follow-up, the discrete-time hazard ratios of $\mathrm{CM}$ onset were $48 \%$ higher for men relative to women $(\mathrm{HR}=1.48 ; p=0.047)$. In each of the remaining two follow-up periods, 9-month and 12-month, the discrete-time hazard ratios of $\mathrm{CM}$ onset were about $104 \%$ higher for men compared to women $(\mathrm{HR}=2.04$; each $p<0.05$, Figure 2$)$. In all models, variables not significantly contributing to the model were removed (race, employment, BMI, GAD-7, and MSS, Supplemental Table 2). Other baseline factors that predicted $\mathrm{CM}$ included increased headache-day frequency ( $\geq 5$ days/month), presence of depression symptoms (PHQ-9 score $\geq 10$ ), and presence of allodynia (ASC score $\geq 3$, Supplemental Table 2).

A loss to follow-up analysis examined the discontinuation profiles of higher risk men and women based on baseline headache frequency, comorbid depression or anxiety, and allodynia. The results indicated that while higher risk men and women with comorbid depression and anxiety are more likely to be lost to follow-up, this effect was more pronounced in men (Supplemental Table 3). Monthly headache frequency was not related to loss to follow-up for men or women. However, for allodynia, there was a significant interaction between men and women in 3-month follow-up only $(p=.042)$ indicating a small but 


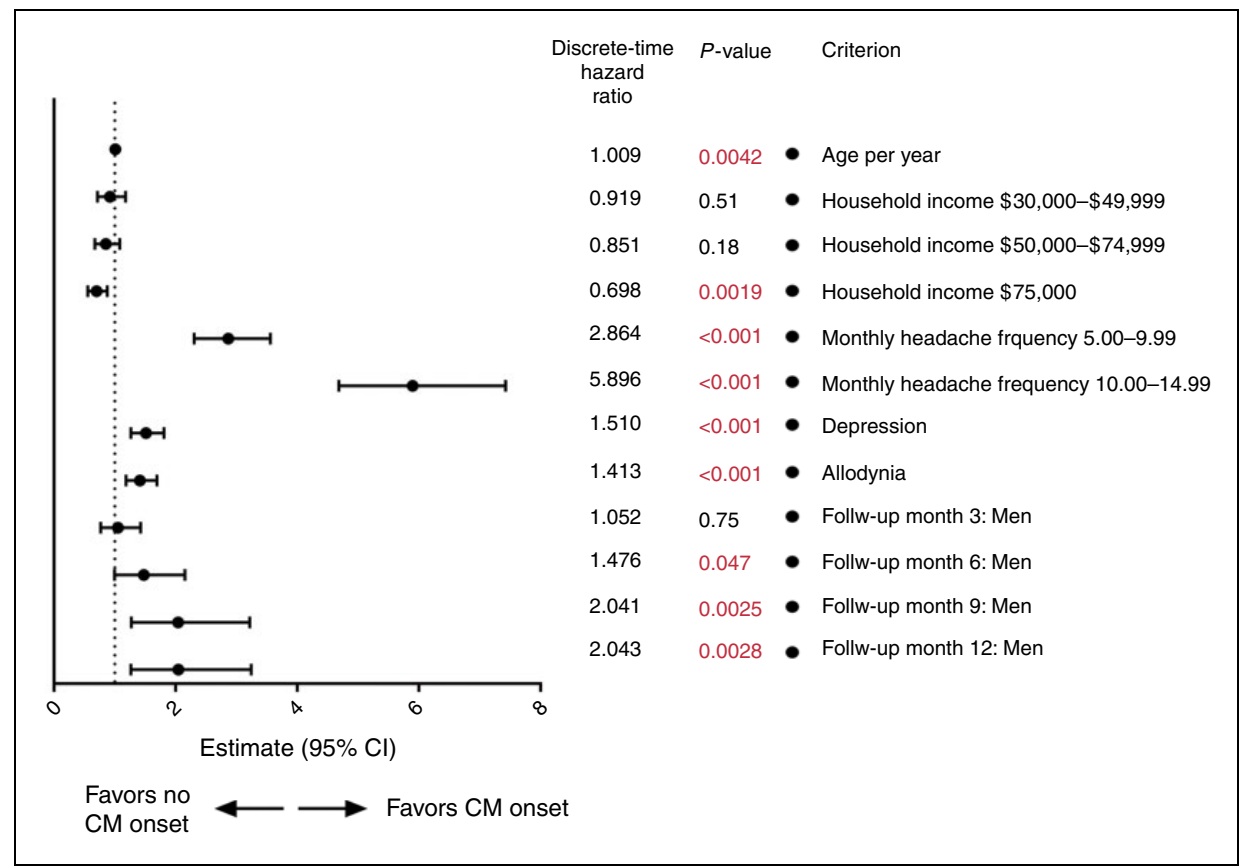

Figure 2. Discrete time hazard model of chronic migraine onset. The model estimates the risk/hazard of CM onset in respondents diagnosed with EM. For example, an estimate of $\mathrm{I} .5$ for depression indicates that respondents with depression at baseline had a $50 \%$ increased risk of CM onset at all follow-up time points compared with respondents without depression after controlling for other factors in the model. This model includes interactions for gender and follow-up period as well as MIDAS and follow-up period. Only the gender interaction is shown in this figure. For full model, see Supplementary Table 2. Significant estimates for the gender interaction indicate that the cumulative incidence of CM was higher in men than women at the 6-month, 9-month, and I-year followup after controlling for other factors.

CM: chronic migraine; EM: episodic migraine; MIDAS: Migraine Disability Assessment.

significant increase in the odds of women with allodynia versus no allodynia (15\% vs. $14 \%$ ) compared to men with allodynia versus no allodynia (12\% vs. $12 \%$ ) being lost to follow-up (Supplemental Table 3).

\section{Discussion}

This analysis of CaMEO Study respondents suggests that, in addition to its well-recognized impact on women, migraine also has a substantial effect on men. Compared with women, men with migraine had a lower attack frequency, less disability, less allodynia, and fewer aura symptoms. Migraine was also less likely to be diagnosed and treated in men compared with women. Differences in aura rates may reflect gender differences in disease expression or differences in symptom reporting (22).

Unexpectedly, a discrete time hazard model of CM onset indicated that, after controlling for known risk factors, men with EM at baseline had a much greater likelihood than women of transitioning to $\mathrm{CM}$ on the 6-, 9-, and 12-month follow-up survey but not at the 3-month follow-up assessment. This phenomenon was further analyzed by performing a loss to follow-up evaluation to determine whether the difference may have been due to selection bias resulting from a greater rate of discontinuation of more severely affected women. However, the results showed that for all follow-ups, while higher-risk men and women with comorbid depression and anxiety were both more likely to discontinue, this effect was more pronounced in men. The greater number of higher-risk men compared with women with the evaluated comorbidities lost to follow-up contradicts the hypothesis of informative censoring contributing to the observed higher risk of CM transition during the timeframe of this study in men. We note that while the presence of allodynia (a negative prognostic factor) was associated with more drop-outs in women than men, this was only evident at the 3-month survey at which men and women were at similar risk of CM transition. Thus, this interaction was not viewed as relevant. Notably, men and women had similar prognoses in the crude models, whereas gender differences only emerged after controlling for other prognostic factors. We can speculate about the reasons for this finding, which could be both biologic and methodologic. One possibility is that there may be differences in prognostic factors between men and women that have not been as extensively examined in men, and therefore were not 
measured and included in our models. Our results indicate that men were more likely than women to use preventives alone $(4.3 \%$ vs. $3.3 \%$, respectively); however, these differences are unlikely to account for the greater likelihood of men transitioning to $\mathrm{CM}$ and could be attributed to confounding by indication. Given that preventive medications reduce headache day frequency in migraine patients, we would expect their use to attenuate, not augment, the higher rate of CM onset in men. We did not have information on head injuries, for example, which are more common in men than women. It is also possible that our measurement of known prognostic factors (e.g. depression, anxiety, headache frequency) was more valid in women than men in this study population, resulting in residual confounding. It is also possible that differences in medical consultation preferences between genders may affect prognosis.

Considered together, the sociodemographic and headache characteristics of men differed from those of women in the present analysis and were largely consistent with the findings of AMPP, which demonstrated a higher prevalence of probable migraine in women compared with men, as well as more severe symptoms, greater headache-related disability and impact, and greater health resource use (4). These gender-specific comorbidity patterns have been further delineated by Teitjen and colleagues, who found a greater prevalence of psychiatric (PHQ-15, psychosocial stress) comorbidities and disability (MIDAS) in women than in men (23). The present study complements other published studies that have also reported differences in migraine comorbidities, characteristics, and impact by gender (5-8,24-26).

The mechanisms underlying the sex-specific differences in the presentation and progression of migraine, which are particularly prominent during the reproductive years (27), have remained elusive but have been linked to differences in the structure and function of the brain and in hormones between men and women. In a mouse model of familial hemiplegic migraine type 1 , spreading depression was modulated by both genetic and hormonal contributions (28). Significant structural differences have been reported between the brains of men and women with migraine, in addition to differences in the involved neural circuitry, which may explain the similar pain intensity but significantly greater perception of pain-related unpleasantness in women compared with men (24). The contribution of hormones to migraine has been recently addressed in studies examining the relationship between migraine and menstruation, which have demonstrated distinct patterns of estrogen reduction during the late luteal phase among women with migraine (29), as well as greater disability and burden in women reporting menstrual-related migraine compared with those indicating that their migraines were unrelated to menstruation (30).
Some limitations of this study should be noted. First, as with all surveys, this study is prone to selection bias; however, by balancing the target sample against key population demographics, steps were taken to minimize such bias (12). Further, the comparability of the CaMEO and AMPP study populations suggests that the data from the CaMEO Study are generalizable to the US migraine population (9). Second, the data are self-reported and not verified by other sources, such as physician diagnoses or medical records, and thus may be subject to recall error. Third, a dichotomous characterization system for EM and CM was applied on a continuum, but the 15-headache days/month cutoff is arbitrary and not fully supported by a biological distinction between the disorders. Indeed, pain duration alone may not be a sufficient classification tool $(31,32)$. Further, headache day frequency varies from month to month; individuals with $\mathrm{CM}$ onset during one 3-month period may have had CM prior to study inclusion and/ or may not have had $\mathrm{CM}$ in a subsequent 3-month period (32). Other factors such as disability and severity may also be useful to consider for pain conditions that are not static and tend to transition between states (i.e. transitioning to and from EM and $\mathrm{CM}$ ) (31). Fourth, because migraine has been studied mostly in women, the covariates and measured prognostic factors may have been more relevant to women than men. Consequently, the statistical models may have been more predictive in women than men, as previously discussed.

Previous studies have reported gender-specific differences in risk factors for renal progression and low back pain chronification $(33,34)$. Consistent with these studies, differences in CM onset in individuals with EM at baseline observed herein between men and women with migraine merit further analyses of the underlying migraine pathophysiology in men compared with women. Future analyses should address gender-specific prognostic patterns in the progression and responses of men and women to migraine treatment and in their rates of remission.

\section{Conclusion}

The current paradigm that migraine is a disease that primarily affects women may contribute to migraine stigma and preclude access by men to accurate diagnosis and appropriate and effective treatment; however, the present findings confirm that migraine can be disabling in men, is less likely to be managed clinically, and further suggest that our understanding of prognostic factors for CM in men may be less well understood. Further analyses in men are warranted to elucidate prognostic factors related to migraine progression and remission as well as reasons for underdiagnosis. 


\section{Article highlights}

- Men with migraine generally have less severe attacks and disability and, if they seek treatment, are less likely to receive a diagnosis than women with migraine.

- Controlling for demographics, headache features, and comorbidities, men had $43 \%$ higher odds of progression from EM to CM at all follow-up periods.

- Prognostic factors may be better understood for women than men.

\section{Acknowledgments}

This study was sponsored by Allergan plc (Dublin, Ireland). Writing and editorial assistance was provided to the authors by Scarlett Geunes-Boyer, PhD, Lee B Hohaia, PharmD, and Dana Franznick, PharmD, of Complete Healthcare Communications, LLC (West Chester, PA), a CHC Group company, and funded by Allergan plc.

\section{Ethical approval}

The CaMEO study was approved by the institutional review board of the Albert Einstein College of Medicine (number 1204-177E).

\section{Declaration of conflicting interests}

The authors declared the following potential conflicts of interest with respect to the research, authorship, and/or publication of this article: Financial arrangements of the authors with companies whose products may be related to the present report are listed below, as declared by the authors. Ann I. Scher, in the past 12 months, has received honoraria from and is an advisory board member for Allergan, receives grant support from CDMRP and CNRM, and is on the editorial boards of Cephalalgia and Pain Medicine. The views expressed are those of the author(s) and do not necessarily reflect the official views of the Uniformed Services University of the Health Sciences or the Department of Defense. Shuu-Jiun Wang, in the past 12 months, has received personal compensation for consulting, advisory boards, or speaker activities from Allergan, GlaxoSmithKline (Taiwan), Pfizer (Taiwan), and Eli Lilly and has received research support from Daiichi-Sankyo, Pfizer (Taiwan), and Eli Lilly. Zaza Katsarava has received personal compensation for consulting, advisory boards, or speaker activities from Allergan, Amgen, Biogen, and Boehringer-Ingelheim. Dawn C Buse, in the past 12 months, has received grant support and honoraria from Allergan, Avanir, Amgen, Eli Lilly and Company, and Promius. She is on the editorial board of Current Pain and Headache Reports, the Journal of Headache and Pain, Pain Medicine News, and Pain Pathways magazine. Kristina M Fanning is an employee of Vedanta Research, which has received support funded by Allergan, Amgen, Dr. Reddy's Laboratories, Eli Lilly, GlaxoSmithKline, Merck \& Co., and Novartis, via grants to the National Headache Foundation. Vedanta has received funding directly from Allergan plc for work in the CaMEO Study. Aubrey Manack Adams is a fulltime employee of Allergan plc and owns stock in the company. Richard B Lipton serves on the editorial boards of Neurology and Cephalalgia and as senior advisor to Headache. He has received research support from the NIH. He also receives support from the Migraine Research Foundation and the National Headache Foundation. He has reviewed for the NIA and NINDS, serves as consultant, advisory board member, or has received honoraria from: Alder, Allergan, Amgen, Autonomic Technologies, Avanir, Boston Scientific, CoLucid, Dr. Reddy's, Electrocore, Eli Lilly, eNeura Therapeutics, GlaxoSmithKlein, Merck, Novartis, Teva, and Vedanta. He receives royalties from Wolff's Headache, 8th Edition, Oxford Press University, 2009 and Informa. He holds stock options in eNeura Therapeutics and Biohaven.

\section{Funding}

The authors disclosed receipt of the following financial support for the research, authorship, and/or publication of this article: This work was supported by Allergan plc.

\section{References}

1. Vos T, Flaxman AD, Naghavi M, et al. Years lived with disability (YLDs) for 1160 sequelae of 289 diseases and injuries 1990-2010: A systematic analysis for the Global Burden of Disease Study 2010. Lancet 2012; 380: 2163-2196.

2. Lillis J, Graham Thomas J, Seng EK, et al. Importance of pain acceptance in relation to headache disability and pain interference in women with migraine and overweight/obesity. Headache 2017; 57: 709-718.

3. Adams AM, Serrano D, Buse DC, et al. The impact of chronic migraine: The Chronic Migraine Epidemiology and Outcomes (CaMEO) Study methods and baseline results. Cephalalgia 2015; 35: 563-578.

4. Buse DC, Loder EW, Gorman JA, et al. Sex differences in the prevalence, symptoms, and associated features of migraine, probable migraine and other severe headache: Results of the American Migraine Prevalence and Prevention (AMPP) Study. Headache 2013; 53: 1278-1299.

5. Victor TW, Hu X, Campbell JC, et al. Migraine prevalence by age and sex in the United States: A life-span study. Cephalalgia 2010; 30: 1065-1072.

6. Lipton RB, Bigal ME, Diamond M, et al. Migraine prevalence, disease burden, and the need for preventive therapy. Neurology 2007; 68: 343-349.

7. Lipton RB, Diamond S, Reed M, et al. Migraine diagnosis and treatment: Results from the American Migraine Study II. Headache 2001; 41: 638-645.

8. Lipton RB, Stewart WF and Simon D. Medical consultation for migraine: Results from the American Migraine Study. Headache 1998; 38: 87-96.

9. Lipton RB, Manack Adams A, Buse DC, et al. A comparison of the Chronic Migraine Epidemiology and Outcomes (CaMEO) Study and American Migraine 
Prevalence and Prevention (AMPP) Study: Demographics and headache-related disability. Headache 2016; 56: 1280-1289.

10. Dodick DW, Loder EW, Manack Adams A, et al. Assessing barriers to chronic migraine consultation, diagnosis, and treatment: Results from the Chronic Migraine Epidemiology and Outcomes (CaMEO) Study. Headache 2016; 56: 821-834.

11. Buse DC, Scher AI, Dodick DW, et al. Impact of migraine on the family: Perspectives of people with migraine and their spouse/domestic partner in the CaMEO Study. Mayo Clin Proc 2016; 91: 596-611.

12. Manack Adams A, Serrano D, Buse DC, et al. The impact of chronic migraine: The Chronic Migraine Epidemiology and Outcomes (CaMEO) Study methods and baseline results. Cephalalgia 2015; 35: 563-578.

13. Scher AI, Buse DC, Fanning KM, et al. Comorbid pain and migraine chronicity: The Chronic Migraine Epidemiology and Outcomes Study. Neurology 2017; 89: 461-468.

14. Stewart WF, Lipton RB, Celentano DD, et al. Prevalence of migraine headache in the United States. Relation to age, income, race, and other sociodemographic factors. JAMA 1992; 267: 64-69.

15. Headache Classification Committee of the International Headache Society. The International Classification of Headache Disorders, 3rd edition (beta version). Cephalalgia 2013; 33: 629-808.

16. Silberstein SD, Lipton RB and Sliwinski M. Classification of daily and near-daily headaches: Field trial of revised IHS criteria. Neurology 1996; 47: 871-875.

17. Silberstein SD, Lipton RB, Solomon S, et al. Classification of daily and near-daily headaches in the headache clinic. Proposed revisions to the International Headache Society criteria. In: Olesen J (ed.) Frontiers in headache research. New York, NY: Raven Press, 1994, pp.117-126.

18. Headache Classification Subcommittee of the International Headache Society. The International Classification of Headache Disorders, 2nd edition. Cephalalgia 2004; 24: 9-160.

19. Bigal ME, Ashina S, Burstein R, et al. Prevalence and characteristics of allodynia in headache sufferers: A population study. Neurology 2008; 70: 1525-1533.

20. Lipton RB, Bigal ME, Ashina S, et al. Cutaneous allodynia in the migraine population. Ann Neurol 2008; 63: $148-158$.
21. R Core Team. R: A language and environment for statistical computing, http://www.R-project.org/ (2017, accessed 26 June 2018).

22. Pisanu C, Preisig M, Castelao E, et al. High leptin levels are associated with migraine with aura. Cephalalgia 2017; 37: 435-441.

23. Tietjen GE, Herial NA, Hardgrove J, et al. Migraine comorbidity constellations. Headache 2007; 47: 857-865.

24. Maleki N, Linnman C, Brawn J, et al. Her versus his migraine: Multiple sex differences in brain function and structure. Brain 2012; 135: 2546-2559.

25. Loewendorf AI, Matynia A, Saribekyan H, et al. Roads less traveled: Sexual dimorphism and mast cell contributions to migraine pathology. Front Immunol 2016; 7: 140. DOI: 10.3389/fimmu.2016.00140.

26. Scheff NN and Gold MS. Sex differences in the inflammatory mediator-induced sensitization of dural afferents. J Neurophysiol 2011; 106: 1662-1668.

27. Vetvik KG and MacGregor EA. Sex differences in the epidemiology, clinical features, and pathophysiology of migraine. Lancet Neurol 2017; 16: 76-87.

28. Eikermann-Haerter K, Dilekoz E, Kudo C, et al. Genetic and hormonal factors modulate spreading depression and transient hemiparesis in mouse models of familial hemiplegic migraine type 1. J Clin Invest 2009; 119: 99-109.

29. Pavlovic JM, Allshouse AA, Santoro NF, et al. Sex hormones in women with and without migraine: Evidence of migraine-specific hormone profiles. Neurology 2016; 87: 49-56.

30. Pavlovic JM, Stewart WF, Bruce CA, et al. Burden of migraine related to menses: Results from the AMPP study. J Headache Pain 2015; 16: 24.

31. Von Korff M and Dunn KM. Chronic pain reconsidered. Pain 2008; 138: 267-276.

32. Serrano D, Lipton RB, Scher AI, et al. Fluctuations in episodic and chronic migraine status over the course of 1 year: Implications for diagnosis, treatment and clinical trial design. J Headache Pain 2017; 18: 101.

33. Chang PY, Chien LN, Lin YF, et al. Risk factors of gender for renal progression in patients with early chronic kidney disease. Medicine (Baltimore) 2016; 95: e4203. DOI: 10.1097/MD.0000000000004203.

34. Lee HI, Lee ST, Kim M, et al. Sex differences in predicting chronicity of low-back pain after acute trauma using lumbar muscle area. Am J Phys Med Rehabil 2015; 94: 123-130. 


\section{DuEPublico}

DE S S S BN R G

\section{Duisburg-Essen Publications online}

Offen im Denken

Ub | universitäts

This text is made available via DuEPublico, the institutional repository of the University of Duisburg-Essen. This version may eventually differ from another version distributed by a commercial publisher.

DOI: $\quad 10.1177 / 0333102418786266$

URN: urn:nbn:de:hbz:464-20210510-152305-5

This publication is with permission of the rights owner freely accessible due to an Alliance licence and a national licence (funded by the DFG, German Research Foundation) respectively.

(C) International Headache Society 2018. All rights reserved. 